\title{
Valutazione di efficienza nella somministrazione dell'ormone della crescita (GH)
}

\author{
Efficiency evaluation in the growth hormone $(\mathrm{GH})$ administration
}

\author{
Federico Spandonaro ${ }^{1}$, Letizia Mancusi ${ }^{2}$ \\ Università di Tor Vergata, Roma \\ 2 4Health Innovation, Roma
}

\begin{abstract}
Treatment with growth hormone (somatropin) is effective in six different medical conditions: growth hormone deficiency (GHD), Turner syndrome (TS), growth retardation in children born small for gestational age (SGA), Prader-Willi syndrome (PWS), growth retardation due to chronic renal insufficiency (CRI), growth retardation associated with a deficiency of the gene SHOX (Short Stature HOmeoboXContaining gene). The treatment proved to be also effective in adults who have an impaired growth hormone (acquired in adulthood or childhood).

The growth hormone $(\mathrm{GH})$ is generally cost effective and, therefore, is usually reimbursed by public health services. In financial terms, GH is a major cost item for health systems. According to the Report OSMED 2010, GH ranks first in Italy between systemic hormonal preparations, excluding sex hormones, distributed by public system, with an annual value of approximately $€ 88$ million ( $+12.7 \%$ compared to 2009 ).

Considering the increasing need to control pharmaceutical expenditure, there is a strong interest for the efficient supply of the hormone by the regional health service. From this point of view, the comparison of the specialties on the market is normally carried out on the basis of the cost per mg; this approach, also used in the main studies of cost-effectiveness, is derived from a logic of cost minimization, but it may still be distorting, not taking into account the efficiency of devices used for the administration of the hormone.

The proposed analysis verifies the efficiency of different available devices, evaluating the potential waste of product, depending both on the device used and on the characteristics of the population exposed to the treatment.

Only in the case of single-dose and disposable formulations is theoretically possible to have zero waste and thus an equivalence between the actual cost of the treatment and the price charged. In other cases, the inefficiency causes a deviation between the actual cost and price. In the latter cases, since the theoretical amount of the population exposed to the treatment, it is estimated that the total share of potentially unused product can vary between $208,000 \mathrm{mg} /$ year to $750,000 \mathrm{mg}$ / year, depending on the device used. In particular, there is an actual average cost of treatment ranging between $+9.9 \%$ and $+11.4 \%$ of the ex-factory price; depending on the different doses and even between the different devices, the difference between the actual price and the theoretical price varies from a minimum of $+6.9 \%$ and a maximum of $+18.7 \%$.
\end{abstract}

\section{Keywords}

Growth hormone deficiency (GHD); Somatropin; Device; Efficiency

\section{INTRODUZIONE}

Il trattamento con ormone della crescita (somatropina) risulta efficace in sei diverse condizioni morbose: deficienza dell'ormone della crescita (GHD), sindrome di Turner (TS), ritardo nella crescita in bambini nati piccoli per l'età gestazionale (SGA), sindrome di Prader-Willi (PWS), ritardo nella crescita dovuta a insufficienza renale cronica (IRC), ritardo nella crescita associata a un deficit del gene SHOX (Short Stature HOmeoboXContaining gene) [1,2]. Il trattamento si è dimostrato inoltre efficace negli adulti che presentino un deficit di ormone della crescita (acquisita in età adulta $\mathrm{o}$ infantile) $[3,4]$.

Le analisi di cost-effectiveness effettuate, ad oggi, concordano sull'accettabilità sociale del trattamento, indipendentemente dalla specialità farmaceutica utilizzata, raccomandandone la rimborsabilità da parte dei terzi paganti; le valutazioni sono fatte in base al costo per "mg" delle specialità farmaceutiche riferito ai centimetri acquistati in altezza o alla qualità della vita guadagnata [5-7].

In Italia sono attualmente presenti sul mercato 7 produttori di GH; in Tabella I si riporta l'elenco delle specialità farmaceutiche autorizzate in Italia (classe di rimborsabilità $\mathrm{A}$ ).
Corresponding author Federico Spandonaro federico.spandonaro@ uniroma2.it

\section{Disclosure}

II presente lavoro è stato supportato da Pfizer Italia 
In termini finanziari, il trattamento rappresenta una voce di costo rilevante per i sistemi sanitari. Secondo quanto riportato nel Rapporto OSMED 2010 con riguardo ai preparati ormonali sistemici, esclusi gli ormoni sessuali, la somatropina, in Italia, è al primo posto per importo fra i farmaci distribuiti dalle strutture pubbliche, per un valore annuo pari a circa 88 milioni di euro, con un incremento del $12,7 \%$ rispetto al 2009 .

In Italia la nota 39 dell'AIFA indica quali debbano essere i criteri clinico-auxologici e di laboratorio per l'autorizzazione al trattamento del paziente con ormone della crescita, sia in età evolutiva, sia in età di transizione 0 in età adulta.

Complessivamente la maggior parte dei soggetti trattati è in età pediatrica e presenta problemi di crescita staturale. Tra questi, il più frequente disturbo endocrino è la ridotta o mancata produzione di livelli sufficienti di ormone della crescita da parte della ghiandola pituitaria (GHD).

In una logica di sempre più stringente necessità di controllo della spesa farmaceutica è cresciuto l'interesse per l'efficienza dell'approvvigionamento dell'ormone da parte dei

\begin{tabular}{|c|c|c|c|}
\hline $\begin{array}{c}\text { Casa } \\
\text { farmaceutica }\end{array}$ & Brand & Confezione & $\mathrm{mg} /$ conf* $^{*}$ \\
\hline \multirow[t]{12}{*}{ Pfizer } & Genotropin & 7 sir 0,6 UI/0,2 mg & 1,4 \\
\hline & Genotropin & $7 \operatorname{sir} 1,2$ UI/0,4 mg & 2,8 \\
\hline & Genotropin & $7 \operatorname{sir} 1,8 \mathrm{UI} / 0,6 \mathrm{mg}$ & 4,2 \\
\hline & Genotropin & 7 sir 2,4 UI/0,8 mg & 5,6 \\
\hline & Genotropin & 7 sir $3 \mathrm{UI} / 1 \mathrm{mg}$ & 7,0 \\
\hline & Genotropin & $4 \operatorname{sir} 3,6 \mathrm{UI} / 1,2 \mathrm{mg}$ & 4,8 \\
\hline & Genotropin & $4 \operatorname{sir} 4,2 \mathrm{UI} / 1,4 \mathrm{mg}$ & 5,6 \\
\hline & Genotropin & $4 \operatorname{sir} 4,8 \mathrm{UI} / 1,6 \mathrm{mg}$ & 6,4 \\
\hline & Genotropin & $4 \operatorname{sir} 5,4 \mathrm{UI} / 1,8 \mathrm{mg}$ & 7,2 \\
\hline & Genotropin & 4 sir 6 Ul/2 mg & 8,0 \\
\hline & Genotropin & $1 \mathrm{fl} 16 \mathrm{Ul} / 5,3 \mathrm{mg}$ & 5,3 \\
\hline & Genotropin & $1 \mathrm{fl} 32 \mathrm{UI} / 12 \mathrm{mg}$ & 12,0 \\
\hline \multirow[t]{2}{*}{ Lilly } & Humatrope & 1 cart $6 \mathrm{mg}$ & 6,0 \\
\hline & Humatrope & 1 cart $12 \mathrm{mg}$ & 12,0 \\
\hline \multirow[t]{2}{*}{ Novo Nordisk } & Norditropin simplex & 1 cart $15 \mathrm{UI} / 5 \mathrm{mg}$ & 5,0 \\
\hline & Norditropin simplex & 1 cart $15 \mathrm{mg}$ & 15,0 \\
\hline Genentech & Nutropin AQ & 1 cart $30 \mathrm{UI} / 10 \mathrm{mg}$ & 10,0 \\
\hline \multirow[t]{2}{*}{ Sandoz } & Omnitrope & $6,7 \mathrm{mg} / \mathrm{ml} 1,5 \mathrm{ml}$ & 10,0 \\
\hline & Omnitrope & $\begin{array}{l}1 \text { cart } 3,3 \mathrm{mg} / \mathrm{ml} \\
1,5 \mathrm{ml}(15 \mathrm{UI})\end{array}$ & 5,0 \\
\hline \multirow[t]{2}{*}{ Merk Serono } & Saizen & $1 \mathrm{fl} 4 \mathrm{UI} / 1,33 \mathrm{mg}$ & 1,3 \\
\hline & Saizen & $1 \mathrm{fl} 24 \mathrm{Ul} / 8 \mathrm{mg}$ & 8,0 \\
\hline Ferring & Zomacton & $1 \mathrm{fl} 12 \mathrm{Ul} / 4 \mathrm{mg}$ & 4,0 \\
\hline
\end{tabular}

Tabella I. Somatropina: farmaci autorizzati in Italia (classe di rimborsabilità A) * II costo ex-factory per mg (netto manovre 2006) è ricompreso tra € 14,58 e € 26,55 cart $=$ cartuccia; $\mathrm{fl}=$ fiala; sir $=$ siringa
Sistemi Sanitari Regionali (SSR). Da questo punto di vista, si osserva che la comparazione delle specialità presenti sul mercato viene, normalmente, effettuata sulla base del costo per $\mathrm{mg}$; tale approccio comparativo, peraltro utilizzato anche nei principali studi di cost-effectiveness, segue una logica di cost-minimization, ma potrebbe risultare comunque distorcente, non tenendo conto dell'efficienza relativa dei devices utilizzati per la somministrazione dell'ormone.

In altri termini, assumere l'equivalenza fra differenziali di costo del trattamento e differenziali di prezzo per mg risulta erroneo, in quanto non tiene conto del fatto che il GH deve essere somministrato per iniezione sottocutanea, mediante utilizzo di appositi devices, i quali determinano livelli diversi di spreco dell'ormone.

Non risulta, tra l'altro, che sia stata effettuata una analisi di costo-efficacia che tenga conto delle diverse tipologie di devices disponibili e quindi del reale costo del trattamento.

Obiettivo del lavoro è, quindi, quello di testare l'efficienza dei devices, con la finalità di verificare quanto gli effettivi costi di trattamento si discostino dai differenziali di prezzo applicati.

\section{METODI}

Sono attualmente disponibili diversi dispositivi, che possono essere suddivisi in due gruppi:

- dispositivi con ago;

- dispositivi senza ago.

Il livello di efficienza dei singoli devices dipende evidentemente dal dosaggio/die della terapia prescritta, nonché dalla stabilità dei prodotti dopo la prima apertura, il che implica un numero massimo di giorni di trattamento possibili, dopo il quale il prodotto scade. L'analisi è stata condotta su quasi tutti i devices in commercio in Italia. Non sono stati presi in considerazione:

- "Saizen flaconi monodose 1,33 mg", in quanto il flacone deve essere ricostituito ogni giorno, e a soluzione ricostituita si consiglia l'uso immediato (la stabilità è dimostrata fino a 24 ore se conservata da $+2 \mathrm{a}+8^{\circ} \mathrm{C}$ ), così che lo "spreco" risulta essere il complemento a 1,33 della dose/ die);

- "Zomacton flacone da 4 mg", in quanto è prevista la ricostituzione con due diverse possibilità di diluizione, che determinano diverse concentrazioni di prodotto e anche diverse somministrazioni - penna senza ago o semplice siringa graduata rendendo non praticabile effettuare i calcoli necessari. 
Il calcolo degli sprechi è stato effettuato per singolo device, a partire dal numero di giornate di trattamento possibili, in funzione del dosaggio/die e della necessaria sostituzione per fine stabilità del prodotto.

Evidentemente, nel caso di formulazioni in monodose e monouso, gli sprechi possono considerarsi nulli.

Ovviamente il calcolo dello spreco riferito al mero aspetto tecnico legato alle caratteristiche del device risulta del tutto teorico: lo spreco effettivo dipende, oltre che dalle caratteristiche del device, anche dalla dose/die prescritta, la quale dipende, nell'età evolutiva, sostanzialmente dalla distribuzione dei pazienti per peso e patologia.

Per stimare quindi gli sprechi effettivi, è necessario conoscere 1'epidemiologia del fenomeno e le caratteristiche della popolazione target.

La conoscenza dell'epidemiologia italiana del fenomeno è alquanto deficitaria, nonostante la Nota 39 dell'AIFA, pena la non rimborsabilità del farmaco da parte del SSN, preveda una sorveglianza epidemiologica stringente, attraverso l'istituzione di Commissioni Regionali ad hoc, e di un Registro Informatizzato nazionale, assegnandone all'Istituto Superiore di Sanità la gestione centralizzata, con l'onere della sorveglianza epidemiologica nazionale.

Per colmare le carenze dei registri, si è quindi provveduto a una stima della popolazione bersaglio in base al "tasso di esposizione al trattamento", separatamente per classi di popolazione: età evolutiva, età di transizione ed età adulta.

Per quanto concerne i pazienti in età evolutiva (0-18 anni), la citata Nota 39 dell'AIFA indica un tasso di esposizione al trattamento pari a 1:2000. Afferma, inoltre, che la coorte di soggetti affetti dalle principali patologie per cui è indicata la terapia con GH debba essere considerata sostanzialmente stabile nel tempo e distribuita in modo omogeneo sul territorio.

Facendo proprie queste indicazioni, e facendo riferimento alla popolazione italiana di età compresa tra 0 e 18 anni residente al $1^{\circ}$ gennaio 2010, per stimare i casi prevalenti per

\begin{tabular}{lccccc}
\hline \multicolumn{1}{c}{ Parametri } & GHD & TS & PWS & SGA & IRC \\
\hline$N^{\circ}$ pazienti all'avvio di trattamento & 7.036 & 2.749 & 485 & 990 & 806 \\
$\mathrm{~N}^{\circ}$ pazienti prossimi all'altezza dell'età adulta & 2.547 & 1.349 & 75 & 127 & 157 \\
Età media a inizio trattamento & 9,14 & 9,30 & 7,42 & 8,18 & 9,00 \\
Età media a fine trattamento & 16,37 & 16,45 & 15,21 & 14,18 & 13,95 \\
Tasso di drop-out al primo anno di trattamento & 0,04 & 0,03 & 0,02 & 0,03 & 0,12 \\
Dose (paz. 0-17 anni media mg/kg/die) & 0,03 & 0,04 & 0,03 & 0,04 & 0,04 \\
\hline
\end{tabular}

Tabella II. Parametri (dal database KIGS). Modificata da [9]

$\mathrm{GHD}=$ deficienza dell'ormone della crescita; IRC = insufficienza renale cronica; PWS = sindrome di Prader-Willi;

$\mathrm{SGA}=$ ritardo nella crescita in bambini nati piccoli per l'età gestazionale; TS = sindrome di Turner

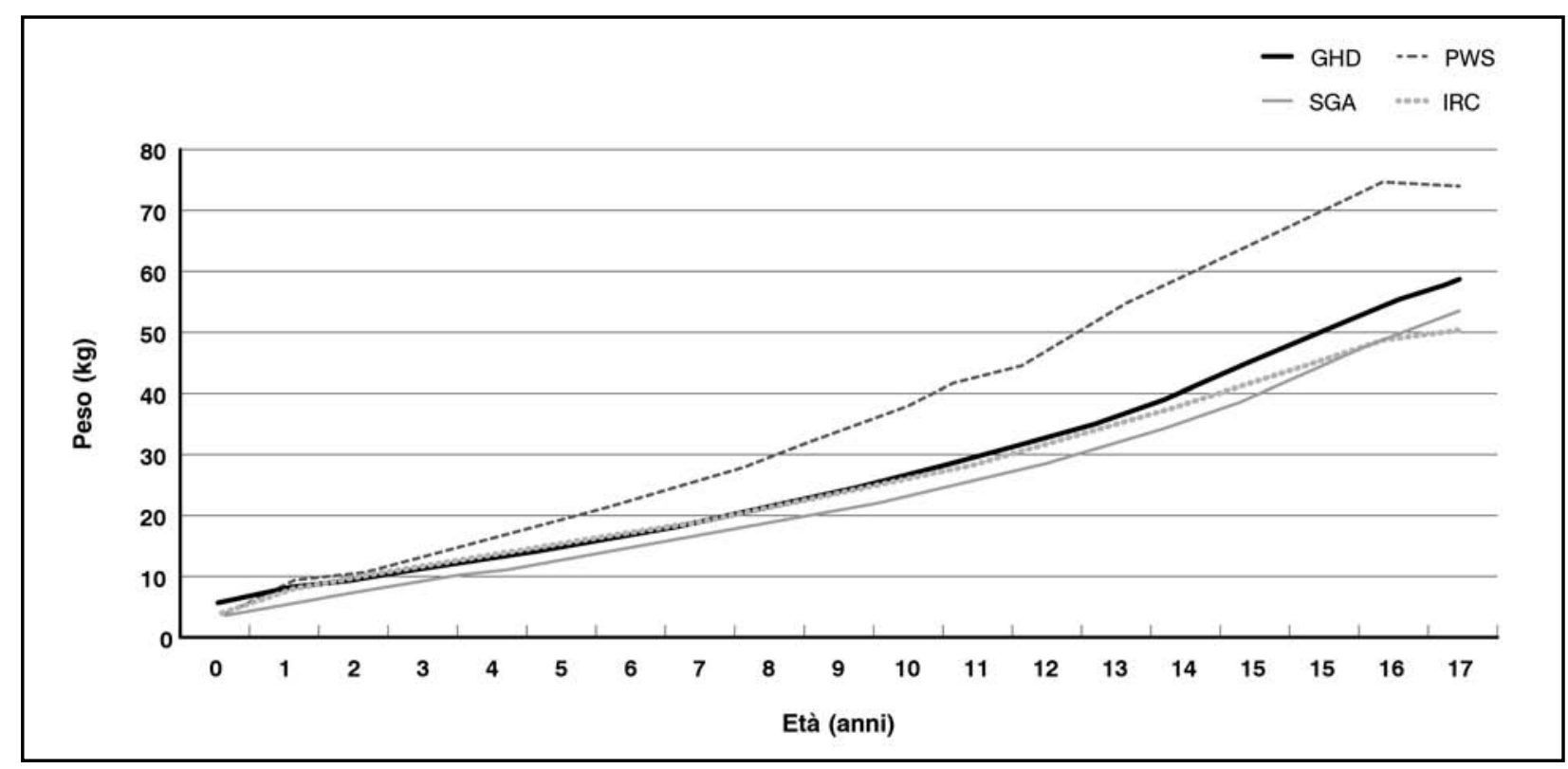

Figura 1. Pazienti in età pediatrica: distribuzione del peso per patologia ed età (maschi). Modificata da [9] 


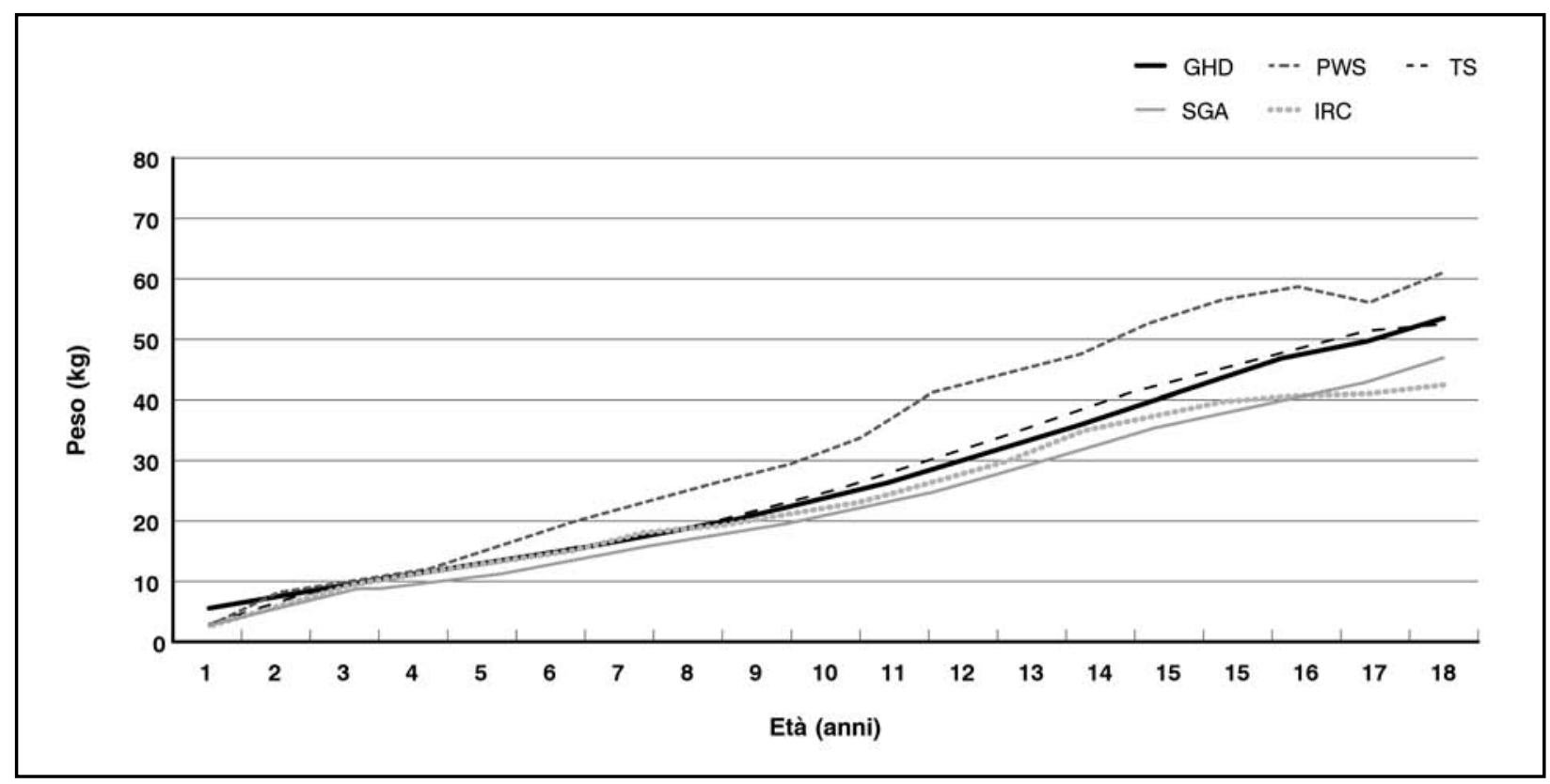

Figura 2. Pazienti in età pediatrica: distribuzione del peso per patologia ed età (femmine). Modificata da [9]

patologia si è utilizzata la ripartizione per patologia relativa ai soggetti in trattamento con GH nel Regno Unito [8]. Per l'ulteriore suddivisione per sesso, nelle singole patologie, si è poi adottata la distribuzione desumibile dal database $\mathrm{KIGS}^{1}$.

La posologia raccomandata di GH varia per diagnosi e anche in funzione della "dimensione" del paziente, questa ultima determinata in base al peso o alla superficie corporea. È stato pertanto necessario, per singola patologia, definire alcuni standard di riferimento riguardo ai parametri essenziali per la determinazione della dose terapeutica/die. In particolare, per ogni patologia con indicazione alla terapia con GH è indispensabile, oltre alla dose terapeutica in $\mathrm{mg} /$ die pro $\mathrm{Kg}$ di peso corporeo, conoscere:

- la distribuzione per sesso;

- la distribuzione del peso per età (e per sesso);

- l'età media di inizio trattamento;

- la durata media del trattamento.

Per quanto riguarda tali parametri, si è fatto riferimento a quanto utilizzato dalla valutazione HTA assunta dal NICE del Regno Unito nel 2011 [9], che si riporta in Tabella II e nelle Figure 1 e 2.

La distribuzione del peso medio per sesso, età e patologia è stata poi confrontata con la distribuzione per età e sesso specifica delle curve di crescita italiane, in particolare con le

KIGS -Kabi International Growth Study (Pfizer Database): è il più grande archivio internazionale di farmacoepidemiologia sull'ormone della crescita (GH), nei bambini e negli adolescenti. E stato istituito nel 1987, ad oggi sono arruolati circa 80.000 pazienti di 52 paesi (http://www.medicaloutcomes. pfizer.com). Accesso: maggio 2012 distribuzioni che si riferiscono al $3^{\circ}$ e $50^{\circ}$ percentile [10]; tale raffronto è stato eseguito al fine di verificare la coerenza e, quindi, la confrontabilità dei dati internazionali e nazionali, specifici per sesso, età e per singola patologia. Si riportano in Figura 3, a titolo esemplificativo, le distribuzioni relative al GHD.

Passando all'età di transizione (18-25 anni), per quanto riguarda i soggetti in trattamento affetti da GHD, si è assunto che siano pari al $34 \%$ dei soggetti trattati in età evolutiva [11]. Considerando, altresì, che in media la durata del trattamento in questi pazienti è pari a circa 7 anni, con un tasso di drop-out al primo anno dello 0,04\% [9] e presupponendo una distribuzione omogenea per età.

Infine, per i soggetti con più di 25 anni con carenza di GH (Adult-onset growth hormone deficiency-AOGHD) si possono distinguere tre grandi gruppi:

- adulti che presentano una carenza di GH dalla nascita o dall'infanzia;

- adulti che hanno sviluppato una carenza di GH in epoca successiva, in seguito a traumi o ad altri tipi di danno della ghiandola che produce il GH stesso (ipofisi); - adulti con carenza di GH idiopatica.

L'AOGHD è prevalentemente causata da tumori pituitari o peripituitari, e dai trattamenti correlati, chirurgici o radioterapici.

Uno studio di popolazione danese effettuato retrospettivamente sui flussi sanitari nazionali (registro tumori, registro nazionale ricoveri ospedalieri, registro delle cause di morte) per gli anni dal 1980 al 1999 [12] ha individuato una coorte di soggetti potenzialmente eleggibili al trattamento con GH. Complessivamen- 


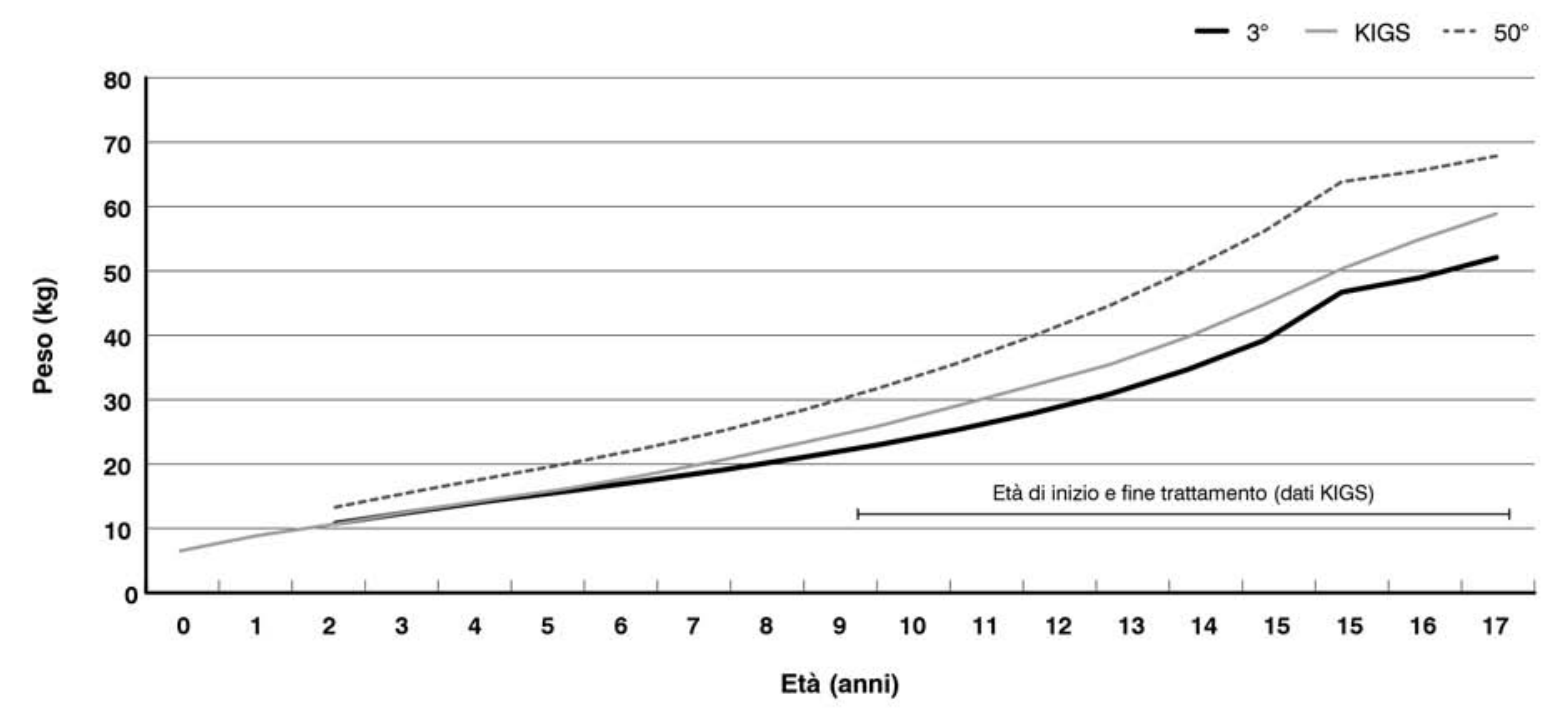

Figura 3. Distribuzione del peso per età in pazienti con GHD in trattamento (maschi)

$3^{\circ}=3^{\circ}$ centile delle curve di crescita italiane [10]; KIGS = Kabi International Growth Study (Pfizer Database):

$50^{\circ}=50^{\circ}$ centile delle curve di crescita italiane [10]

te sono stati identificati, nei 20 anni suddetti, 1.329 soggetti adulti (età > 18 anni), 744 maschi e 585 femmine. L'età media alla diagnosi risulta pari a 51,3 anni nei maschi e 49,8 nelle femmine. Il tasso di incidenza media risulta pari a 1,90 per 100.000 abitanti over 18 nei maschi e di 1,42 per 100.000 abitanti nelle femmine.

\section{RISULTATI}

Per quanto concerne l'aspetto degli sprechi dei device, il risultato delle elaborazioni può essere espresso in forma sintetica, mediante un "indice di efficienza" (IE) per singolo device: l'indice è ottenuto come rapporto tra $\mathrm{i}$ consumi annui effettivi di GH generati da uno specifico device e la dose terapeutica an-

\begin{tabular}{|c|c|c|c|c|c|c|c|}
\hline $\begin{array}{l}\text { Dose/die } \\
\text { (mg) }\end{array}$ & $\begin{array}{l}\text { Dose anno } \\
\text { (mg) }\end{array}$ & $\begin{array}{c}\text { Cartuccia } \\
5 \mathrm{mg}\end{array}$ & $\begin{array}{c}\text { Cartuccia } \\
6 \mathrm{mg}\end{array}$ & $\begin{array}{c}\text { Cartuccia } \\
8 \mathrm{mg}\end{array}$ & $\begin{array}{c}\text { Cartuccia } \\
10 \mathrm{mg}\end{array}$ & $\begin{array}{c}\text { Tubofiala } \\
\text { cartuccia } 12 \mathrm{mg}\end{array}$ & $\begin{array}{c}\text { Tubofiala } \\
\text { cartuccia } 15 \mathrm{mg}\end{array}$ \\
\hline 0,2 & 73,0 & 1,00 & $1,07^{\star}$ & $1,43^{*}$ & $1,78^{*}$ & $2,14^{\star}$ & $2,67^{\star}$ \\
\hline 0,3 & 109,5 & 1,04 & 1,00 & 1,03 & $1,19 *$ & $1,43^{*}$ & $1,78^{*}$ \\
\hline 0,4 & 146,0 & 1,04 & 1,00 & 1,00 & 1,00 & $1,07^{*}$ & $1,34^{*}$ \\
\hline 0,5 & 182,5 & 1,00 & 1,00 & 1,00 & 1,00 & 1,00 & $1,07^{\star}$ \\
\hline 0,6 & 219,0 & 1,04 & 1,00 & 1,03 & 1,04 & 1,00 & 1,00 \\
\hline 0,7 & 255,5 & 1,02 & 1,07 & 1,04 & 1,02 & 1,01 & 1,02 \\
\hline 0,8 & 292,0 & 1,04 & 1,07 & 1,00 & 1,04 & 1,00 & 1,04 \\
\hline 0,9 & 328,5 & 1,11 & 1,11 & 1,11 & 1,01 & 1,03 & 1,04 \\
\hline 1,0 & 365,0 & 1,00 & 1,00 & 1,00 & 1,00 & 1,00 & 1,00 \\
\hline 1,1 & 401,5 & 1,14 & 1,09 & 1,04 & 1,01 & 1,09 & 1,05 \\
\hline 1,2 & 438,0 & 1,04 & 1,00 & 1,11 & 1,04 & 1,00 & 1,04 \\
\hline 1,3 & 474,5 & 1,28 & 1,15 & 1,03 & 1,10 & 1,03 & 1,05 \\
\hline 1,4 & 511,0 & 1,19 & 1,07 & 1,14 & 1,02 & 1,07 & 1,07 \\
\hline 1,5 & 547,5 & 1,11 & 1,00 & 1,07 & 1,11 & 1,00 & 1,00 \\
\hline 1,6 & 584,0 & 1,04 & 1,25 & 1,00 & 1,04 & 1,07 & 1,04 \\
\hline 1,7 & 620,5 & 1,47 & 1,18 & 1,18 & 1,18 & 1,01 & 1,10 \\
\hline 1,8 & 657,0 & 1,39 & 1,11 & 1,11 & 1,11 & 1,11 & 1,04 \\
\hline 1,9 & 693,5 & 1,31 & 1,05 & 1,05 & 1,05 & 1,05 & 1,13 \\
\hline \multirow[t]{2}{*}{2,0} & 730,0 & 1,25 & 1,00 & 1,00 & 1,00 & 1,00 & 1,07 \\
\hline & & 1,13 & 1,06 & 1,07 & 1,09 & 1,11 & 1,19 \\
\hline
\end{tabular}

Tabella III. Indice di efficienza (IE) per dimensione device

II calcolo è effettuato per singolo device e per singola dose/die secondo la seguente formula: IE $=[($ dose/die $\times 365)+($ spreco/anno $)] /($ dose $/$ die $\times 365)$ * Spreco dovuto a sostituzione per fine stabilità del prodotto 


\begin{tabular}{|c|c|c|c|c|c|c|c|c|}
\hline $\begin{array}{c}\text { Area } \\
\text { geografica }\end{array}$ & $\begin{array}{c}\text { Residenti } 2010 \\
0-18 \text { anni }\end{array}$ & $\begin{array}{c}\text { Esposti al } \\
\text { trattamento* }\end{array}$ & $\begin{array}{c}\text { GHD } \\
(57,4 \%)\end{array}$ & $\begin{array}{c}\text { TS } \\
(18,7 \%)\end{array}$ & $\begin{array}{l}\text { PWS } \\
(4,6 \%)\end{array}$ & $\begin{array}{c}\text { SGA } \\
(5,2 \%)\end{array}$ & $\begin{array}{c}\text { IRC } \\
(2,5 \%)\end{array}$ & $\begin{array}{c}\text { Altro } \# \\
(11,6 \%)\end{array}$ \\
\hline Italia & 10.831 .152 & 5.416 & 3.109 & 1.013 & 249 & 282 & 135 & 628 \\
\hline Nord & 4.705 .411 & 2.353 & 1.350 & 440 & 108 & 122 & 59 & 273 \\
\hline Nord-Ovest & 2.708 .819 & 1.354 & 777 & 253 & 62 & 70 & 34 & 157 \\
\hline Nord-Est & 1.996 .592 & 998 & 573 & 187 & 46 & 52 & 25 & 116 \\
\hline Centro & 2.011 .523 & 1.006 & 577 & 188 & 46 & 52 & 25 & 117 \\
\hline Sud e Isole & 4.114 .218 & 2.057 & 1.181 & 385 & 95 & 107 & 51 & 239 \\
\hline Sud & 2.827 .126 & 1.414 & 811 & 264 & 65 & 74 & 35 & 164 \\
\hline Isole & 1.287 .092 & 644 & 369 & 120 & 30 & 33 & 16 & 75 \\
\hline
\end{tabular}

Tabella IV. Stima dei soggetti in età pediatrica in trattamento per patologia e area geografica (elaborazione degli Autori su dati Istat) * tasso di esposizione al trattamento 1:2000

\# i dati si riferiscono ai soggetti in età evolutiva trattati nel Regno Unito nel 2006 [8] (nell'articolo si fa riferimento a "indicazioni non autorizzate" - nel 2006 in UK non risulta autorizzata la SHOX)

$\mathrm{GHD}=$ deficienza dell'ormone della crescita; IRC = insufficienza renale cronica; PWS = sindrome di Prader-Willi;

$\mathrm{SGA}=$ ritardo nella crescita in bambini nati piccoli per l'età gestazionale; TS = sindrome di Turner

\begin{tabular}{|c|c|c|c|c|c|c|c|c|c|c|c|}
\hline \multirow{2}{*}{$\begin{array}{c}\text { Area } \\
\text { geografica }\end{array}$} & \multicolumn{2}{|c|}{ GHD } & \multirow{2}{*}{$\begin{array}{c}\text { TS } \\
\mathbf{F}\end{array}$} & \multicolumn{2}{|c|}{ PWS } & \multicolumn{2}{|c|}{ SGA } & \multicolumn{2}{|c|}{ IRC } & \multicolumn{2}{|c|}{ Altro } \\
\hline & $\begin{array}{c}M \\
(70 \%)\end{array}$ & $\begin{array}{c}F \\
(30 \%)\end{array}$ & & $\begin{array}{c}M \\
(50 \%)\end{array}$ & $\begin{array}{c}F \\
(50 \%)\end{array}$ & $\begin{array}{c}M \\
(60 \%)\end{array}$ & $\begin{array}{c}F \\
(40 \%)\end{array}$ & $\begin{array}{c}M \\
(71 \%)\end{array}$ & $\begin{array}{c}F \\
(29 \%)\end{array}$ & $\begin{array}{c}M \\
(50 \%)\end{array}$ & $\begin{array}{c}F \\
(50 \%)\end{array}$ \\
\hline Italia & 2.176 & 933 & 1.013 & 125 & 125 & 169 & 113 & 96 & 39 & 314 & 314 \\
\hline Nord & 945 & 405 & 440 & 54 & 54 & 73 & 49 & 42 & 17 & 136 & 136 \\
\hline Nord-Ovest & 544 & 233 & 253 & 31 & 31 & 42 & 28 & 24 & 10 & 79 & 79 \\
\hline Nord-Est & 401 & 172 & 187 & 23 & 23 & 31 & 21 & 18 & 7 & 58 & 58 \\
\hline Centro & 404 & 173 & 188 & 23 & 23 & 31 & 21 & 18 & 7 & 58 & 58 \\
\hline Sud e Isole & 827 & 354 & 385 & 47 & 47 & 64 & 43 & 37 & 15 & 119 & 119 \\
\hline Sud & 568 & 243 & 264 & 33 & 33 & 44 & 29 & 25 & 10 & 82 & 82 \\
\hline Isole & 259 & 111 & 120 & 15 & 15 & 20 & 13 & 11 & 5 & 37 & 37 \\
\hline
\end{tabular}

Tabella V. Stima dei soggetti in età pediatrica in trattamento per patologia e sesso (elaborazione degli Autori su dati KIGS [9])

nua teoricamente prevista, presupponendo una dose/die invariante nell'anno. Un valore dell'indice pari ad 1 equivale quindi all'as-

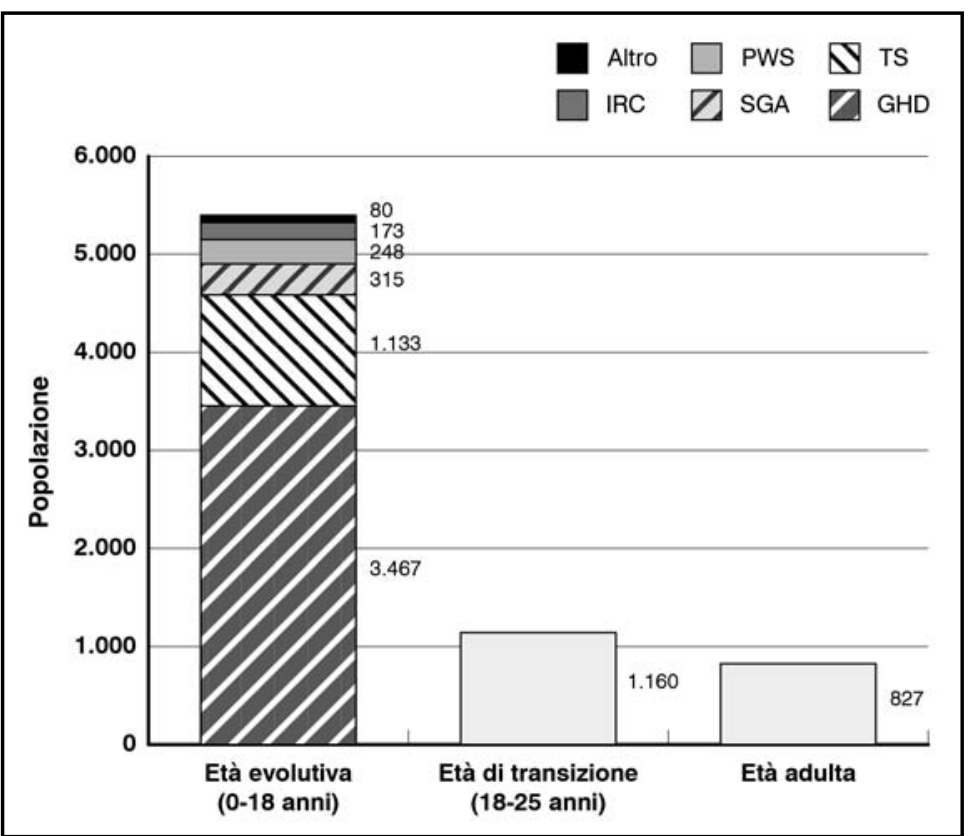

Figura 4. Distribuzione della popolazione prevalente in trattamento stimata per classi di età e patologia senza di spreco, mentre un valore superiore esprime in termini percentuali lo spreco; ciò premesso, per specifiche prescrizioni di dose/ die, si può arrivare anche a sprechi teorici di oltre il $250 \%$ (Tabella III).

Tale evidenza, come precedentemente argomentato, è stata applicata a una stima della popolazione bersaglio, specifica per età, sesso, patologia, e dove necessario tenendo conto della distribuzione per peso.

Per quanto concerne i soggetti in età evolutiva, proiettando sulla popolazione italiana il tasso di esposizione così come indicato nella nota 39 dell'AIFA, la distribuzione per patologia del Regno Unito, nonché le evidenze del database KIGS per quanto concerne la suddivisione fra i sessi, si arriva a una stima complessiva di 5.416 individui (Tabella IV), di cui 2.880 maschi (Tabella V).

Relativamente ai dosaggi per età e patologia applicati, le ipotesi adottate sono le seguenti:

- Età evolutiva dosaggi minimi:

- GHD 0,023 mg/kg/die;

- TS $0,045 \mathrm{mg} / \mathrm{kg} / \mathrm{die}$;

- PWS 0,035 mg/kg/die;

- SGA 0,035 mg/kg/die;

- IRC $0,035 \mathrm{mg} / \mathrm{kg} / \mathrm{die}$; 
- Età evolutiva dosaggi massimi:

- GHD 0,039 mg/kg/die;

- TS $0,050 \mathrm{mg} / \mathrm{kg} / \mathrm{die}$;

- PWS 0,035 mg/kg/die;

- SGA 0,035 mg/kg/die;

- IRC $0,050 \mathrm{mg} / \mathrm{kg} / \mathrm{die}$.

Non potendosi procedere a simulazioni relative a tutte le possibili combinazioni, considerando inoltre che la scelta del dosaggio (non esistendo un dosaggio standard) presuppone una valutazione clinica su singolo caso in base alla risposta soggettiva al trattamento, nonché alla scienza e coscienza del clinico, si è ritenuto che il valore minimo e massimo indicato nella review di Kirk [13] ricomprenda tutti i possibili scenari intermedi.

Per quanto concerne l'età di transizione, sulla base delle ipotesi descritte precedentemente, si giunge a una stima di circa 1.160 soggetti in trattamento tra i 18 e i 25 anni, di cui 812 maschi.

Infine, per la popolazione adulta, la proiezione dei dati danesi sulla popolazione italiana adulta al $1^{\circ}$ gennaio 2010 evidenzia un numero presumibile di 827 soggetti eleggibili alla terapia con $\mathrm{GH}$, di cui 457 maschi.

Nei soggetti in età di transizione e in età adulta, la posologia non è più strettamente

\begin{tabular}{lcccc}
\hline $\begin{array}{c}\text { Area } \\
\text { geografica }\end{array}$ & $\begin{array}{c}\text { Età evolutiva } \\
\text { (0-18 anni) }\end{array}$ & $\begin{array}{c}\text { Età di transizione } \\
\text { (18-25 } \text { anni) }\end{array}$ & $\begin{array}{c}\text { Età } \\
\text { adulta }\end{array}$ & Totale \\
\hline Italia & 5.416 & 1.160 & 827 & 7.403 \\
Nord & 2.353 & 504 & 382 & 3.239 \\
Nord-Ovest & 1.354 & 290 & 222 & 1.866 \\
Nord-Est & 998 & 214 & 160 & 1.372 \\
Centro & 1.006 & 215 & 164 & 1.386 \\
Sud e Isole & 2.057 & 441 & 281 & 2.778 \\
Sud & 1.414 & 303 & 190 & 1.907 \\
Isole & 644 & 138 & 91 & 873 \\
\hline
\end{tabular}

Tabella VI. Stima dei soggetti in trattamento con GH per classi di età e per area geografica

legata al peso del paziente, ma la strategia terapeutica prevede, generalmente, l'avvio del trattamento a dosaggi più bassi di quelli utilizzati in età pediatrica con graduali incrementi, fino alla determinazione della dose minima giornaliera che permetta una normalizzazione dei livelli sierici di IGF-I (all'interno del range di normalità per sesso ed età), senza evidenza di effetti collaterali. Le dosi di partenza possono essere, in base all'età del paziente, schematizzate come segue [3]:

\begin{tabular}{|c|c|c|c|c|c|c|c|}
\hline Brand & Confezione & mg/conf. & $\begin{array}{c}\text { Età } \\
\text { evolutiva }\end{array}$ & $\begin{array}{l}\text { Età di } \\
\text { transizione }\end{array}$ & $\begin{array}{c}\text { Età } \\
\text { adulta }\end{array}$ & Totale & $\begin{array}{l}\text { Indice di } \\
\text { efficienza }\end{array}$ \\
\hline \multicolumn{2}{|c|}{ Consumo teorico annuo $(\mathrm{mg})$} & & 2.584 .273 & 338.720 & 97.017 & 3.020 .010 & \\
\hline \multicolumn{8}{|c|}{ Consumo effettivo annuo (mg) } \\
\hline \multirow[t]{12}{*}{ Genotropin } & 7 sir 0,6 Ul/0,2 mg & 1,4 & \multirow{10}{*}{2.188 .467} & \multirow{10}{*}{338.720} & \multirow{10}{*}{97.017} & \multirow{10}{*}{2.624 .204} & \multirow{10}{*}{0,87} \\
\hline & $7 \operatorname{sir} 1,2 \mathrm{UI} / 0,4 \mathrm{mg}$ & 2,8 & & & & & \\
\hline & 7 sir 1,8 UI/0,6 mg & 4,2 & & & & & \\
\hline & $7 \operatorname{sir} 2,4 \mathrm{UI} / 0,8 \mathrm{mg}$ & 5,6 & & & & & \\
\hline & 7 sir $3 \mathrm{UI} / 1 \mathrm{mg}$ & 7,0 & & & & & \\
\hline & $4 \operatorname{sir} 3,6 \mathrm{UI} / 1,2 \mathrm{mg}$ & 4,8 & & & & & \\
\hline & $4 \operatorname{sir} 4,2 \mathrm{UI} / 1,4 \mathrm{mg}$ & 5,6 & & & & & \\
\hline & $4 \operatorname{sir} 4,8 \mathrm{UI} / 1,6 \mathrm{mg}$ & 6,4 & & & & & \\
\hline & $4 \operatorname{sir} 5,4 \mathrm{UI} / 1,8 \mathrm{mg}$ & 7,2 & & & & & \\
\hline & $4 \operatorname{sir} 6 \mathrm{UI} / 2 \mathrm{mg}$ & 8,0 & & & & & \\
\hline & $1 \mathrm{fl} 16 \mathrm{UI} / 5,3 \mathrm{mg}$ & 5,3 & 2.981 .852 & 374.007 & 98.878 & 3.454 .737 & 1,14 \\
\hline & $1 \mathrm{fl} 32 \mathrm{Ul} / 12 \mathrm{mg}$ & 12,0 & 2.681 .855 & 338.720 & 207.895 & 3.228 .470 & 1,07 \\
\hline \multirow[t]{2}{*}{ Humatrope } & 1 cart $6 \mathrm{mg}$ & 6,0 & 2.900 .744 & 362.918 & 103.941 & 3.367 .602 & 1,12 \\
\hline & 1 cart 12 mg & 12,0 & 2.681 .855 & 338.720 & 207.895 & 3.228 .470 & 1,07 \\
\hline \multirow{2}{*}{$\begin{array}{l}\text { Norditropin } \\
\text { simplex }\end{array}$} & 1 cart $15 \mathrm{UI} / 5 \mathrm{mg}$ & 5,0 & 2.940 .514 & 352.837 & 97.017 & 3.390 .368 & 1,12 \\
\hline & 1 cart $15 \mathrm{mg}$ & 15,0 & 2.713 .992 & 352.837 & 259.873 & 3.326 .701 & 1,10 \\
\hline Nutropin AQ & 1 cart $30 \mathrm{UI} / 10 \mathrm{mg}$ & 10,0 & 2.732 .929 & 352.837 & 173.248 & 3.259 .015 & 1,08 \\
\hline \multirow[t]{2}{*}{ Omnitrope } & 1 cart $3,3 \mathrm{mg} / \mathrm{ml} \mathrm{1,5 \textrm {ml }}$ & 5,0 & 2.940 .514 & 352.837 & 97.017 & 3.390 .368 & 1,12 \\
\hline & $6,7 \mathrm{mg} / \mathrm{ml} 1,5 \mathrm{ml}$ & 10,0 & 2.732 .929 & 352.837 & 173.248 & 3.259 .015 & 1,08 \\
\hline Saizen & $1 \mathrm{fl} 24 \mathrm{Ul} / 8 \mathrm{mg}$ & 8,0 & 2.795 .099 & 338.720 & 138.601 & 3.272 .420 & 1,08 \\
\hline
\end{tabular}

Tabella VII. Consumi teorici ed effettivi annui in Italia: dose $\mathrm{mg} /$ die minima cart $=$ cartuccia; $\mathrm{fl}=$ fiala; sir $=$ siringa 
- età $<30$ anni: $0,4-0,5 \mathrm{mg} /$ die (la dose può essere più elevata per i pazienti con pregresso trattamento in età pediatrica);

- età 30-60 anni: $0,2-0,3 \mathrm{mg} / \mathrm{die}$;

- età $>60$ anni: $0,1-0,2 \mathrm{mg} / \mathrm{die}$

Tale schema terapeutico prevede nei controlli successivi (a 1-2 mesi) incrementi della dose di $0,1-0,2 \mathrm{mg} /$ die in base alla risposta clinica (livelli sierici di IGF-I, effetti collaterali, considerazioni individuali, quali l'intolleranza al glucosio). Intervalli temporali più lunghi e incrementi di dose più piccoli possono essere necessari nei pazienti anziani.

Per i pazienti in età di transizione, avendo nel modello proposto ipotizzato per tutti l'avvio del trattamento in età pediatrica, per le simulazioni di consumo si utilizza un dosaggio medio a regime, superiore ed equivalente a circa la metà dell'ultima dose/die assunta in età pediatrica, minimo $0,8 \mathrm{mg} /$ die massimo $1,0 \mathrm{mg} /$ die [14].

Per i soggetti in età adulta eleggibili al trattamento con GH, non è stato possibile determinare in letteratura un'indicazione sulla durata media del trattamento. Si presuppone che, essendo una terapia sostitutiva, essa debba essere proseguita, sine finem, a meno di eventuali effetti collaterali o eventi av- versi riconducibili al trattamento stesso, o ad eventuali condizioni patologiche insorte. La simulazione dei consumi è effettuata presupponendo un dosaggio medio in questi soggetti compreso tra $0,2 \mathrm{mg} / \mathrm{die}$ e $0,3 \mathrm{mg} / \mathrm{die}$.

Si riporta in Tabella VI il riepilogo dei soggetti in trattamento stimati, per classi di età $\mathrm{e}$ per area geografica; complessivamente i soggetti esposti al trattamento risultano 7.403, con una prevalenza sulla popolazione italiana al $1^{\circ}$ gennaio 2010 , pari a 1,23 per 10.000 abitanti. In Figura 4 si riporta la distribuzione per età e per patologia della popolazione in trattamento.

In Tabella VII si evidenziano i consumi teorici ed effettivi annui per tipo di device, riferiti alla popolazione prevalente sopra descritta, assumendo il dosaggio in $\mathrm{mg} /$ die minimo; in Tabella VIII si riportano gli stessi dati, assumendo per i medesimi pazienti il dosaggio in $\mathrm{mg} / \mathrm{die}$ massimo.

Per quanto riguarda i devices monodose/ monouso, i consumi annui effettivi nell'età evolutiva non corrispondono esattamente al consumo annuo teorico, in quanto sono stati decurtati i consumi relativi ai pazienti che, in relazione all'età e quindi al peso medio cor-

\begin{tabular}{|c|c|c|c|c|c|c|c|}
\hline Brand & Confezione & mg/conf. & $\begin{array}{c}\text { Età } \\
\text { evolutiva }\end{array}$ & $\begin{array}{c}\text { Età di } \\
\text { transizione }\end{array}$ & $\begin{array}{c}\text { Età } \\
\text { adulta }\end{array}$ & Totale & $\begin{array}{l}\text { Indice di } \\
\text { efficienza }\end{array}$ \\
\hline \multicolumn{2}{|c|}{ Consumo teorico annuo (mg) } & & 3.448 .155 & 423.400 & 145.526 & 4.017 .081 & \\
\hline \multicolumn{3}{|c|}{ Consumo effettivo annuo (mg) } & & & & & \\
\hline \multirow[t]{12}{*}{ Genotropin } & 7 sir 0,6 Ul/0,2 mg & 1,4 & \multirow{10}{*}{2.547 .919} & \multirow{10}{*}{423.400} & \multirow{10}{*}{145.526} & \multirow{10}{*}{3.116 .845} & \multirow{10}{*}{0,78} \\
\hline & $7 \operatorname{sir} 1,2 \mathrm{UI} / 0,4 \mathrm{mg}$ & 2,8 & & & & & \\
\hline & $7 \operatorname{sir} 1,8 \mathrm{UI} / 0,6 \mathrm{mg}$ & 4,2 & & & & & \\
\hline & $7 \operatorname{sir} 2,4 \mathrm{UI} / 0,8 \mathrm{mg}$ & 5,6 & & & & & \\
\hline & $7 \operatorname{sir} 3 \mathrm{UI} / 1,0 \mathrm{mg}$ & 7 & & & & & \\
\hline & $4 \operatorname{sir} 3,6 \mathrm{UI} / 1,2 \mathrm{mg}$ & 4,8 & & & & & \\
\hline & $4 \operatorname{sir} 4,2 \mathrm{UI} / 1,4 \mathrm{mg}$ & 5,6 & & & & & \\
\hline & $4 \operatorname{sir} 4,8 \mathrm{UI} / 1,6 \mathrm{mg}$ & 6,4 & & & & & \\
\hline & $4 \operatorname{sir} 5,4 \mathrm{UI} / 1,8 \mathrm{mg}$ & 7,2 & & & & & \\
\hline & $4 \operatorname{sir} 6 \mathrm{UI} / 2 \mathrm{mg}$ & 8,0 & & & & & \\
\hline & $1 \mathrm{fl} 16 \mathrm{UI} / 5,3 \mathrm{mg}$ & 5,3 & 4.081 .390 & 448.804 & 151.227 & 4.681 .421 & 1,17 \\
\hline & $1 \mathrm{fl} 32 \mathrm{UI} / 12 \mathrm{mg}$ & 12 & 3.651 .275 & 423.400 & 207.895 & 4.282 .570 & 1,07 \\
\hline \multirow[t]{2}{*}{ Humatrope } & 1 cart $6 \mathrm{mg}$ & 6 & 3.932 .526 & 423.400 & 145.526 & 4.501 .451 & 1,12 \\
\hline & 1 cart 12 mg & 12 & 3.651 .275 & 423.400 & 207.895 & 4.282 .570 & 1,07 \\
\hline \multirow{2}{*}{$\begin{array}{l}\text { Norditropin } \\
\text { simplex }\end{array}$} & 1 cart $15 \mathrm{UI} / 5 \mathrm{mg}$ & 5 & 4.194 .988 & 423.400 & 151.586 & 4.769 .974 & 1,19 \\
\hline & 1 cart $15 \mathrm{mg}$ & 15 & 3.651 .975 & 423.400 & 259.873 & 4.335 .248 & 1,08 \\
\hline Nutropin AQ & 1 cart $30 \mathrm{UI} / 10 \mathrm{mg}$ & 10 & 3.758 .431 & 423.400 & 173.248 & 4.355 .080 & 1,08 \\
\hline \multirow[t]{2}{*}{ Omnitrope } & 1 cart 3,3 mg/ml 1,5ml & 5,0 & 4.194 .988 & 423.400 & 151.586 & 4.769 .974 & 1,19 \\
\hline & $6,7 \mathrm{mg} / \mathrm{ml} 1,5 \mathrm{ml}$ & 10,0 & 3.758 .431 & 423.400 & 173.248 & 4.355 .080 & 1,08 \\
\hline Saizen & $1 \mathrm{fl} 24 \mathrm{Ul} / 8 \mathrm{mg}$ & 8 & 3.833 .177 & 423.400 & 149.260 & 4.405 .837 & 1,10 \\
\hline
\end{tabular}

Tabella VIII. Consumi teorici ed effettivi annui in Italia: dose $\mathrm{mg} /$ die massima cart $=$ cartuccia; $\mathrm{fl}=$ fiala; sir $=$ siringa 


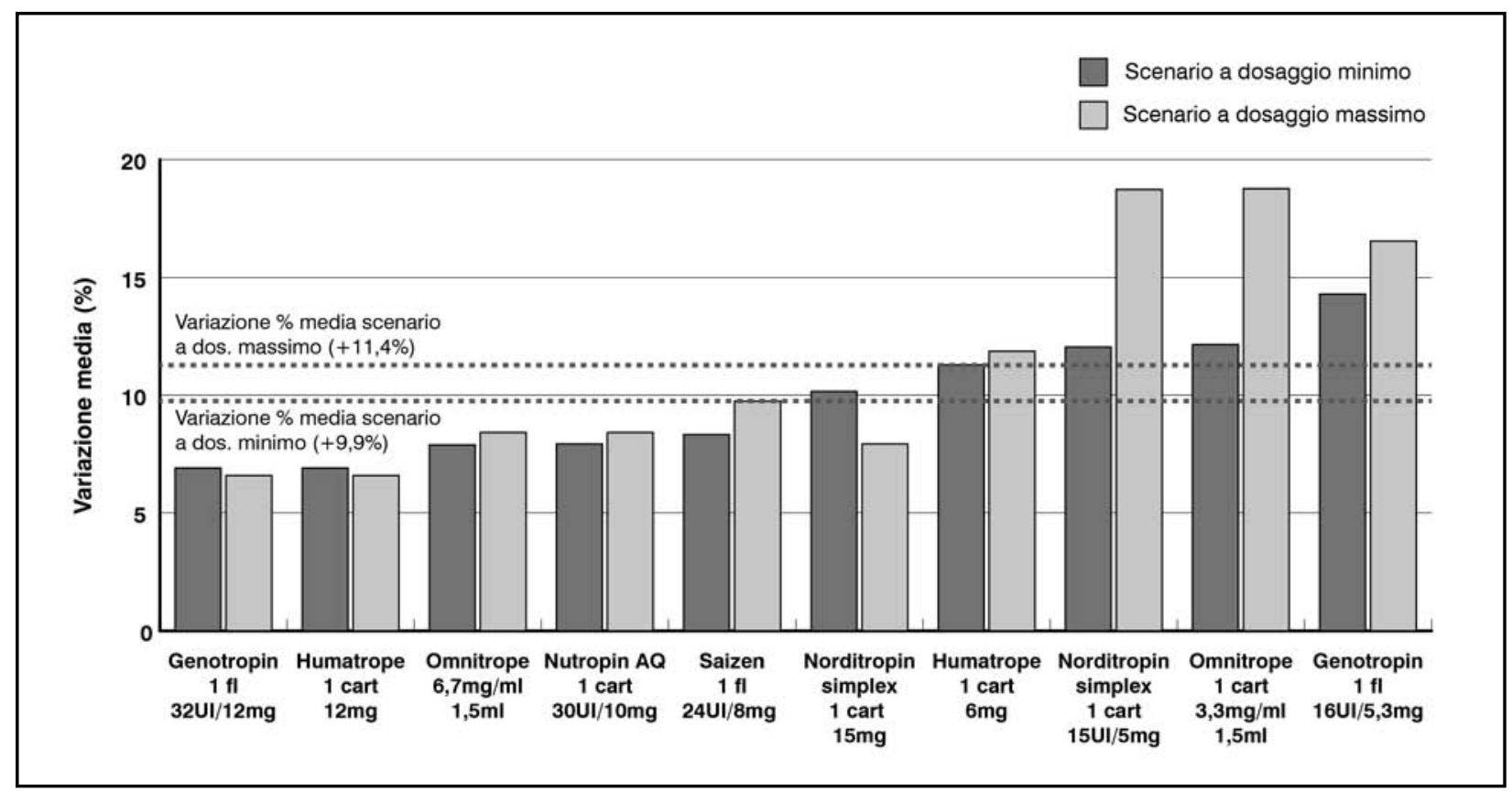

Figura 5. Costo teorico per mg vs costo effettivo per mg (variazione \%)

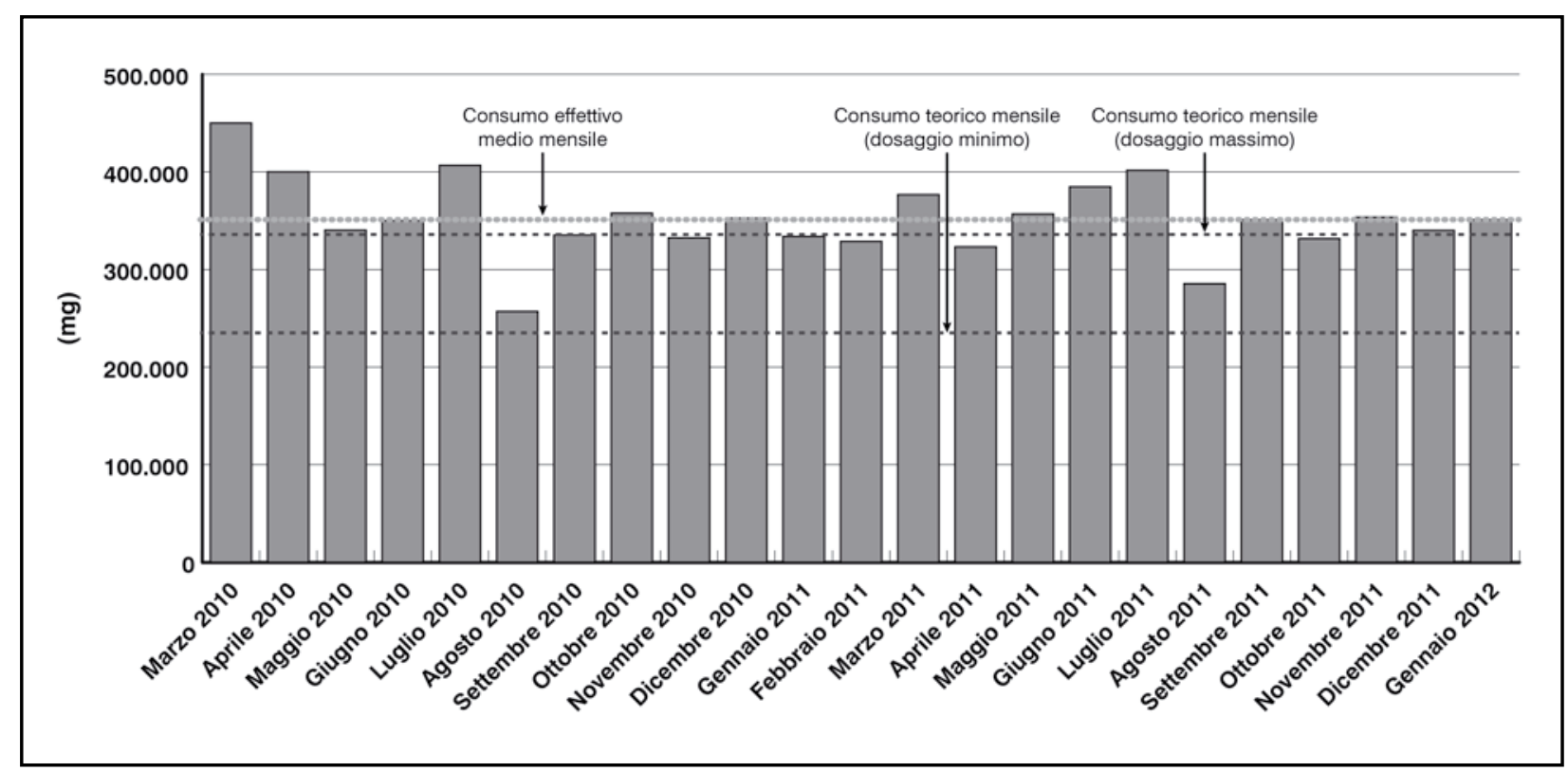

Figura 6. Andamento mensile dei consumi di somatropina vs consumi teorici stimati

poreo, superavano la dose di $2 \mathrm{mg} / \mathrm{die}$, non disponibile.

Pertanto, l'indice di efficienza relativo ai devices monodose/monouso diventa la capacità di copertura del mercato teorico a spreco nullo.

Per una valutazione complessiva, il più verosimile possibile allo scenario italiano, è necessario altresì tenere conto che solo Genotropin e Omnitrope hanno ad oggi indicazione per la PWS.

Per questa patologia, prevista in entrambi gli scenari ipotizzati con un solo dosaggio $(0,035 \mathrm{mg} / \mathrm{kg} / \mathrm{die})$, il consumo teorico annuo è pari a $288.313 \mathrm{mg} /$ annui, equivalenti al $9,6 \%$ e al $7,5 \%$ dei consumi teorici totali, rispettivamente nei dosaggi minimi e massimi ipotizzati, consumi che andrebbero sottratti agli altri brand, che potrebbero pertanto coprire al più il $90,4 \%$ e il $92,5 \%$.

\section{DISCUSSIONE}

Il prezzo ex-factory in mg evidenzia un prezzo medio teorico, per i devices considerati nell'analisi, di $€ 22,44$, con un range di variabilità compreso tra $€ 14,58$ del biosimilare Omnitrope e un massimo di $€ 26,55$ al mg per Genotropin (confezione da 5,3 mg). 
Per quanto riguarda quest'ultimo, sono disponibili formulazioni monodose/monouso, i cui prezzi ex-factory variano da un minimo di $€ 21,89$ per la formulazione $0,6 \mathrm{mg} / \mathrm{die}$ a un massimo di $€ 25,17$ per la formulazione 2 $\mathrm{mg} /$ die. Per questi ultimi devices, fatta salva la quota di mercato con dosaggio die maggiore di $2 \mathrm{mg}$ (sopra precisata), non determinandosi alcuno spreco, il costo effettivo del trattamento coincide con quello desumibile dal prezzo praticato.

Per le altre specialità, come riportato in Figura 5, l'inefficienza determinata dagli sprechi, induce un costo effettivo maggiore; in particolare si evidenzia, a seconda dei casi, un incremento medio sul prezzo ex-factory del $+9,9 \%$ con un minimo del $+6,9 \%$ e un massimo del $+14,4 \%$, nello scenario che presuppone i dosaggi minimi. Nell'ipotesi dell'utilizzo dei dosaggi massimi, l'incremento medio sul prezzo ex-factory al mg risulta del $+11,4 \%$ con un minimo del $+6,6 \%$ e un massimo del $+18,7 \%$.

La proiezione dei consumi medi mensili teorici in "mg" è confrontata in Figura 6 ai dati reali di consumo italiani, marzo 2010-gennaio 2012. I consumi attesi secondo le ipotesi formulate sono inferiori a quelli reali del $6 \%$ nel caso dei dosaggi massimi e del $29 \%$ nel caso dei dosaggi minimi.

Complessivamente, data la stima della popolazione esposta al trattamento, la quota di prodotto inutilizzata risulta variabile tra 208.000 $\mathrm{mg} / \mathrm{anno}$ a $750.000 \mathrm{mg} / \mathrm{anno}$ a seconda del device utilizzato. Si può quindi concludere che le valutazioni comparative effettuate in base al prezzo per mg risultano significativamente distorte dalla mancata considerazione della diversa efficienza dei devices utilizzati e che anche le analisi di costo-efficacia sono affette da un potenziale bias per le medesime ragioni.

\section{BIBLIOGRAFIA}

1. NICE technology appraisal guidance 188. Human growth hormone (somatropin) for the treatment of growth failure in children, 2010

2. Growth Hormone Research Society. Consensus guidelines for the diagnosis and treatment of growth hormone (GH) deficiency in childhood and adolescence: summary statement of the GH research society. J Clin Endocrinol Metab 2000; 85: 3990-3; http://dx.doi.org/10.1210/jc.85.11.3990

3. Cook DM, Yuen KC, Biller BM, et al. American Association of Clinical Endocrinologists medical guidelines for clinical practice for growth hormone use in growth hormone-deficient adults and transition patients - 2009 update. Endocr Pract 2009; 15 (suppl 2):1-29

4. Growth Hormone Research Society. Consensus guidelines for the diagnosis and treatment of adults with growth hormone deficiency: summary statement of the Growth Hormone Research Society Workshop on Adult Growth Hormone Deficiency. J Clin Endocrinol Metab 1998; 83: 379-381; http://dx.doi.org/10.1210/jc.83.2.379

5. Li H, Banerjee S, Dunfield L, et al. Recombinant human growth hormone for treatment of Turner Syndrome: systematic review and economic evaluation [Technology report number 96]. Ottawa: Canadian Agency for Drugs and Technologies in Health (CADTH); 2007

6. Joshi AV, Munro V, Russell MW. Cost-utility of somatropin (rDNA origin) in the treatment of growth hormone deficiency in children. Curr Med Res Opin 2006; 22: 351-7; http://dx.doi.org/10.1185/030079906X80503

7. d'Andon A, Barré S, Hamers F, et al. L'hormone de la croissance chez l'enfant non déficitaire. Evaluation du service rendu à la collectivité. HAS / Service Evaluation des Médicaments et Service Evaluation Economique et Santé Publique, 2011: p. 48, 126-133, 174-181, 203-211, 239-245, 257-258

8. Kirk J, Clayton P. Specialist services and transitional care in paediatric endocrinology in the UK and Ireland. Clin Endocrinol (Oxf) 2006; 65: 59-63; http://dx.doi.org/10.1111/j.1365-2265.2006.02546.x

9. Takeda A, Cooper K, Bird A, et al. Recombinant human growth hormone for the treatment of growth disorders in children: a systematic review and economic evaluation. Health Technol Assess 2010; 14: 1-209, iii-iv. http://dx.doi. org/10.3310/hta14420

10. Cacciari E, Milani S, Balsamo A, et al. Italian cross-sectional growth charts for height, weight and BMI (2 to 29 yr). J Endocrinol Invest 2006; 29: 581-93

11. Bonfig W, Bechtold S, Bachmann S, et al. Reassessment of the optimal growth hormone cut-off level in insulin tolerance testing for growth hormone secretion in patients with childhood-onset growth hormone deficiency during transition to adulthood. J Pediatr Endocrinol Metab 2008; 21: 1049-56 
12. Stochholm K, Juul S, Juel K, et al. Prevalence, incidence, diagnostic delay, and mortality in Turner syndrome. $J$ Clin Endocrinol Metab 2006; 91: 3897-902; http://dx.doi.org/10.1210/jc.2006-0558

13. Kirk J. Indications for growth hormone therapy in children. Arch Dis Child 2012; 97: 63-8; http://dx.doi.org/10.1136/ archdischild-2012-301885.152; http://dx.doi.org/10.1136/adc.2010.186205; http://dx.doi.org/10.1136/archdischild-2012-301885.153

14. Cook DM, Rose SR. A review of guidelines for use of growth hormone in pediatric and transition patients. Pituitary 2012; 15: 301-10; http://dx.doi.org/10.1007/s11102-011-0372-6 